\title{
(6) OPEN ACCESS \\ Socioeconomic status, comorbidity and mortality in patients with type 2 diabetes mellitus in Scotland 2004-2011: a cohort study
}

\author{
Jeremy Walker, Nynke Halbesma, Nazir Lone, David McAllister, Christopher J Weir, \\ Sarah H Wild, on behalf of the Scottish Diabetes Research Network Epidemiology \\ Group
}

Centre for Population Health Sciences, The University of Edinburgh, Edinburgh, UK

\section{Correspondence to} Dr Jeremy Walker, Centre for Population Health Sciences, The University of Edinburgh, Medical School, Teviot Place, Edinburgh EH8 9AG, UK; jeremy.walker@ed.ac.uk

Received 25 September 2015 Revised 16 November 2015 Accepted 29 November 2015 Published Online First 17 December 2015

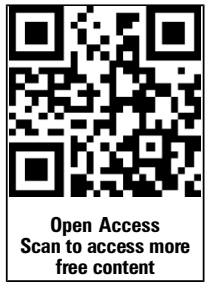

\section{ABSTRACT}

Background Mortality in people with and without diabetes often exhibits marked social patterning, risk of death being greater in deprived groups. This may reflect deprivation-related differences in comorbid disease (conditions additional to diabetes itself). This study sought to determine whether the social patterning of mortality in a population with type 2 diabetes mellitus (T2DM) is explained by differential comorbidity.

Methods Hospital records for 70197 men and 56451 women diagnosed with T2DM at 25 years of age and above in Scotland during the period 2004-2011 were used to construct comorbidity histories. Sex-specific logistic models were fitted to predict mortality at 1 year after diagnosis with T2DM, predicted initially by age and socioeconomic status (SES) then extended to incorporate in turn 5 representations of comorbidity (including the Charlson Index). The capacity of comorbidity to explain social mortality gradients was assessed by observing the change in regression coefficients for SES following the addition of comorbidity.

Results After adjustment for age and Charlson Index, the $O R$ for the contrast between the least deprived and most deprived quintiles of SES for men was $0.79(95 \%$ $\mathrm{Cl} 0.67$ to 0.94 ). For women, the OR was 0.81 (0.67 to 0.97). Similar results were obtained for the 4 other comorbidity measures used.

Conclusions The social patterning of mortality in people with T2DM is not fully explained by differing levels of comorbid disease additional to T2DM itself. Other dimensions of deprivation are implicated in the elevated death rates observed in deprived groups of people with T2DM.

\section{INTRODUCTION}

Mortality in people with diabetes is often elevated relative to non-diabetic populations; a recent meta-analysis concluded that mortality in diabetic populations is increased approximately twofold relative to those without diabetes. ${ }^{1}$ Death from cardiovascular disease is a major contributor to this excess mortality. ${ }^{2}{ }^{3}$ Within diabetic populations, mortality often exhibits social patterning, all-cause mortality risk being raised in more deprived groups. ${ }^{4-6}$ Because many clinical conditions in addition to diabetes demonstrate social patterning, the apparent influence of socioeconomic status (SES) on mortality in diabetic groups may be wholly or partly explained by different burdens of comorbidity (diseases additional to diabetes itself). Assessing the role of comorbidity in the association between SES and mortality is challenging, not least because no generally recognised standard method of measuring comorbidity exists. Representations of comorbidity developed by Charlson et $a l^{7}$ and Elixhauser $e t a l^{8}$ have been used in a variety of contexts. Other approaches to measuring comorbidity include those based on the number of hospital bed-days accrued by the patient, ${ }^{9}$ or the total number of distinct drug classes recorded in the individual's medication history. ${ }^{10} \mathrm{~A}$ number of studies have compared the respective performances of multiple comorbidity measures in predicting mortality, ${ }^{11-13}$ but no measure has emerged as delivering an unequivocal 'best' predictive performance. Consequently, when attempting to determine whether the social mortality gradient observed in people with diabetes is explained by comorbidity, multiple representations of the latter must be assessed to ensure that the findings are not unduly influenced by some peculiarity of an individual comorbidity index. The present study sought to determine whether comorbidity (measured in five different ways) explained social mortality differentials in a population with type 2 diabetes mellitus (T2DM).

\section{METHODS}

\section{Data sources}

Data for people diagnosed with T2DM in Scotland were obtained from the Scottish Care Information-Diabetes Collaboration (SCI-DC) data set, ${ }^{14}$ which stores clinical and demographic data on almost all patients with diabetes in Scotland. The study was based on an extract of SCI-DC data performed in 2011, and was restricted to people diagnosed with T2DM at 25 years of age and above during the period from 1 January 2004 to 18 May 2011. The study end date was defined as 18 May 2012, ensuring that 1 year of follow-up time was available for all patients. The SCI-DC data were linked to hospital discharge records, national death records and prescribing data by the Information Services Division (ISD) of National Health Service (NHS) National Services Scotland (NHSNSS). Generation of the linked data set was approved by the responsible ethics committee, Caldicott guardians and the NHSNSS Privacy Application Committee (application no. 33/11). All data were anonymised before release to the researchers. 


\section{Comorbidity and SES}

Hospital discharge records covering the period 5 years prior to diagnosis with T2DM were used to construct four different measures of comorbidity. Scottish hospital records include six diagnosis fields (one holding the clinical condition mainly responsible for admission, the remaining five storing details of conditions which coexist or develop during the episode of healthcare and affect the management of the patient). The presence of a relevant clinical condition in any of these six diagnosis fields was accepted in constructing the comorbidity measures used in this study. For the period under investigation, all conditions were coded in accordance with the International Classification of Diseases, V.10 (ICD10). ${ }^{15}$ The first comorbidity measure derived from hospital records was the Charlson Comorbidity Index (CCI $)^{7}$ as refined by Romano et al. ${ }^{16}$ This is a single-number (integer) quantity with possible values ranging from 0 (no comorbidity) to 29 (maximum comorbidity). Mapping of the original Charlson groups to ICD10 codes followed the scheme used by ISD to construct hospital standardised mortality ratios. ${ }^{17}$ The second representation of comorbidity was a set of binary indicators marking the presence or absence of 30 specific disease groups defined by Elixhauser et $a .^{8}$ In constructing these indicators, the mapping of Elixhauser's original comorbidities to ICD10 provided by Quan et $a l^{18}$ was used. The third comorbidity measure constructed was the number of hospital in-patient bed-days accrued by each person during the 5 -year period prior to diagnosis with T2DM. To provide a fourth measure, a group of binary indicators marking the presence or absence of 25 disease groups defined by ISD to guide coding of comorbid clinical conditions on Scottish hospital discharge records ${ }^{19}$ was created. These groups were developed by condensing 1127 ICD10 codes from 232 categories, and represent conditions deemed to have substantial prognostic significance. A fifth comorbidity measure was derived from patients' prescribing histories, namely a count of the number of unique drug classes (British National Formulary (BNF) $\operatorname{codes}^{20}$ ) present in the database of medication prescribed in primary care during the 5 years before T2DM diagnosis.

The measure of SES used was the Scottish Index of Multiple Deprivation (SIMD). ${ }^{21}$ This is a small area-based ranked measure which combines 38 indicators of deprivation across seven different conceptual domains. Development of SIMD is sponsored by the Scottish Government via a rigorous academic process as the standard method of assessing deprivation in Scotland. It is the only measure of SES for which up-to-date national data covering the whole Scottish population are routinely available, and was thus the natural choice for this population-based study. SIMD is a ranked (as distinct from an absolute) measure and, for this reason, cannot be represented in analysis as a numeric quantity; rather, it must be included as some kind of categorical variable. For the purposes of this investigation, rankings of the 6505 geographical areas recorded in SIMD were expressed as quintiles (the first and fifth quintiles representing the most and least deprived groups in the population, respectively). This reduction to a five-way classification scheme offered a good compromise between sacrificing too much information (too few levels) and ending up with undesirably small numbers in some strata (too many levels).

\section{Statistical analysis}

An initial logistic regression model was fitted in which mortality at 1 year after T2DM diagnosis (ie, vital status dead vs alive) was predicted only by age at diagnosis (represented by a restricted cubic spline function to accommodate non-linear effects of age on mortality) and by SES (binary indicators marking quintiles $2-5$ of SIMD, with the most deprived quintile 1 defined as the reference category). Separate models were fitted for each sex, to accommodate potential sex-related differences in the prognostic implications of some disease groups (notably cancer, which includes malignancies at sex-specific sites eg, prostate, cervix). From these initial models, the parameter estimates representing the effect of SIMD quintile on 1-year mortality were noted. Modelling was performed using a combination of R software (V.3.0.3; the 'lrm' function in package 'rms') and SAS (V.9.4; the 'LOGISTIC' procedure). The models were then extended by adding each of the five comorbidity measures in turn (only a single measure being included at any one time), and recording the amended estimates for the effect of SIMD on mortality. In fitting the models with comorbidity, certain manipulations of the comorbidity measures were performed in the interests of analytic practicality; these are now summarised for each measure involved. First, the distribution of the CCI was found to be heavily skewed, with high proportions of zero values, that is, no comorbidity (men: $80.5 \%$; women: $80.4 \%$ ). Reflecting this, the original scores on the CCI were reduced to a four-way categorical scheme: $0,1,2-3$, and 4 and above (conceptualised as representing respectively zero, mild, moderate and high levels of comorbidity). Values of the CCI discretised in this way were found to exhibit material interactions with age, so terms representing the interaction of CCI with the spline components of age were included in the models. A second set of manipulations was motivated by problems of non-convergence experienced when fitting the logistic model which featured the Elixhauser comorbidity set. These difficulties were attributable to the indicator marking the presence of AIDS, which exhibited extremely low prevalence $(<0.01 \%)$. Convergence difficulties also arose in connection with a small number of other 'rare' Elixhauser comorbidities, for example, blood loss anaemia (prevalence $<0.05 \%$ in men). To permit successful modelling of the Elixhauser comorbidities, the indicator representing AIDS was omitted from the models: this was justified on the grounds that because the condition was so rare in the cohort, its effect on overall mortality was likely to be extremely small. To permit meaningful parameter estimates to be obtained for other sparsely represented conditions in the Elixhauser set, Firth's bias-reducing penalised likelihood method $^{22}$ was adopted. A third series of modifications to the original comorbidity measure values was applied in connection with in-patient bed-days. The distribution of this was found to be heavily skewed with a small number of extremely large values, and was therefore reduced for analysis to the four-way ordinal classification used by Parks et $a l^{9}$ : $0,1-4,5-10$, and 11 and above. A fourth manipulation of comorbidity values was performed when fitting models which included the ISD comorbidity suite: here, the indicator representing the presence of AIDS/HIV was again omitted in the interests of permitting the logistic model to converge. Finally, values for the prescribing-based comorbidity measure (number of distinct BNF codes) showed a non-linear relationship with the outcome (1-year mortality), so this measure was represented in analysis as a restricted cubic spline function. In summary, the comorbidity measures as represented in the models consisted of two ordinal schemes (CCI and number of bed-days), two groups of condition-specific binary indicators (Elixhauser with 29 retained indicators and the ISD scheme with 24 retained indicators) and one continuous (more correctly, non-negative integer) quantity (number of unique BNF codes), represented as a spline function. 


\section{RESULTS}

From an initial candidate cohort of 126725 (representing all patients diagnosed with T2DM in Scotland at ages 25 and above during the study period), 77 (0.06\%) were excluded because date of death was uncertain. The cohort retained for analysis consisted of 70197 men and 56451 women. Characteristics of the study cohort are summarised in table 1.

The prevalence of individual comorbidities in the two indicator-based comorbidity measures (Elixhauser and the ISD comorbidity scheme) is shown in table 2 . Summary values for the two ordinal comorbidity schemes (CCI and number of bed-days) and the single continuous quantity (number of unique BNF codes) are given in table 3 .

Figure 1 shows the effect of SES (quintile of SIMD) on 1-year mortality in men, corresponding estimates for women being presented in figure 2. These charts illustrate the OR estimate for each quintile of SIMD (relative to Q1, the most deprived quintile) as a predictor of 1-year mortality, adjusted for age only and for age plus each of the five comorbidity measures. For men, the OR estimate for the contrast between SIMD quintile 5 (least deprived) and quintile 1 (most deprived) was 0.70 (95\% CI 0.59 to 0.83 ) before adjustment for comorbidity. After adjustment for comorbidity, corresponding values were 0.79 (0.67 to 0.94 ) for the CCI; 0.77 ( 0.65 to 0.92$)$ for the Elixhauser indicators; 0.77 (0.65 to 0.91$)$ for in-patient bed-days; 0.79 (0.66 to 0.94 ) for the ISD comorbidity set; and 0.75 (0.63 to 0.88$)$ for number of unique drug classes. For women, corresponding estimates were 0.74 (0.62 to 0.89$)$ for no comorbidity; 0.81 ( 0.67 to 0.97$)$ for the CCI; 0.77 (0.64 to 0.93$)$ for the Elixhauser indicators; 0.81 (0.68 to 0.97$)$ for in-patient bed-days; 0.77 ( 0.64 to 0.93$)$ for the ISD comorbidity set; and 0.81 ( 0.67 to 0.97 ) for number of unique drug classes.

\section{DISCUSSION}

The pervasive nature of SES-related health inequalities (including mortality gradients) is now universally accepted, although debate continues as to the explanation(s) for this ubiquitous phenomenon. $^{23}$ Because differential mortality by SES is observed in many societies, populations and contexts, it is arguably not surprising that such an association has also been recorded in people with diabetes. However, although 'there are important indicators of social deprivation which predict mortality over and above diabetic health status itself' (ref. ${ }^{4}$, p.205), the present study is believed to be the first to investigate

Table 1 Characteristics of study cohort

\begin{tabular}{lcc}
\hline & Men $(\mathbf{n}=\mathbf{7 0}$ 197) & Women $(\mathbf{n}=56$ 451) \\
\hline $\begin{array}{l}\text { Age at diabetes diagnosis, years } \\
\text { (mean (SD)) }\end{array}$ & $60.5(12.3)$ & $63.6(13.0)$ \\
SIMD quintile: $\mathrm{n}(\%)$ in & & \\
Q1 (most deprived) & $15567(22.2)$ & $13851(24.5)$ \\
Q2 & $15474(22.0)$ & $13275(23.5)$ \\
Q3 & $14374(20.5)$ & $11432(20.3)$ \\
Q4 & $13536(19.3)$ & $10042(17.8)$ \\
Q5 (least deprived) & $11246(16.0)$ & $7851(13.9)$ \\
$\mathrm{n}$ (\%) dead within 1 year after diabetes diagnosis in & \\
SIMD Q1 & $371(2.4)$ & $363(2.6)$ \\
Q2 & $393(2.5)$ & $319(2.4)$ \\
Q3 & $327(2.3)$ & $282(2.5)$ \\
Q4 & $304(2.2)$ & $271(2.7)$ \\
Q5 & $231(2.1)$ & $198(2.5)$ \\
\hline
\end{tabular}

Table 2 Prevalence of individual comorbidities represented in the Elixhauser and Information Services Division (ISD) comorbidity schemes

\begin{tabular}{|c|c|c|c|c|}
\hline \multirow[b]{3}{*}{ Comorbidity } & \multicolumn{4}{|c|}{ Elixhauser } \\
\hline & \multicolumn{2}{|l|}{ Men } & \multicolumn{2}{|c|}{ Women } \\
\hline & $\mathbf{n}$ & Per cent & $\mathbf{N}$ & Per cent \\
\hline Congestive heart failure & 2289 & 3.26 & 1451 & 2.57 \\
\hline Cardiac arrhythmias & 3554 & 5.06 & 2875 & 5.09 \\
\hline Valvular disease & 898 & 1.28 & 841 & 1.49 \\
\hline Pulmonary circulation disorders & 410 & 0.58 & 423 & 0.75 \\
\hline Peripheral vascular disorders & 1564 & 2.23 & 806 & 1.43 \\
\hline Hypertension & 7843 & 11.17 & 7410 & 13.13 \\
\hline Paralysis & 314 & 0.45 & 228 & 0.40 \\
\hline Other neurological disorders & 930 & 1.32 & 821 & 1.45 \\
\hline Chronic pulmonary disease & 3194 & 4.55 & 3568 & 6.32 \\
\hline Diabetes, uncomplicated & 2593 & 3.69 & 2021 & 3.58 \\
\hline Diabetes, complicated & 71 & 0.10 & 41 & 0.07 \\
\hline Hypothyroidism & 308 & 0.44 & 1330 & 2.36 \\
\hline Renal failure & 782 & 1.11 & 580 & 1.03 \\
\hline Liver disease & 909 & 1.29 & 617 & 1.09 \\
\hline Peptic ulcer excluding bleeding & 611 & 0.87 & 452 & 0.80 \\
\hline AIDS* & 6 & 0.01 & 1 & 0.00 \\
\hline Lymphoma & 183 & 0.26 & 100 & 0.18 \\
\hline Metastatic cancer & 270 & 0.38 & 391 & 0.69 \\
\hline Solid tumour without metastasis & 1680 & 2.39 & 1621 & 2.87 \\
\hline $\begin{array}{l}\text { Rheumatoid arthritis/collagen vascular } \\
\text { disease }\end{array}$ & 384 & 0.55 & 727 & 1.29 \\
\hline Coagulopathy & 221 & 0.31 & 166 & 0.29 \\
\hline Obesity & 1307 & 1.86 & 1353 & 2.40 \\
\hline Weight loss & 250 & 0.36 & 242 & 0.43 \\
\hline Fluid and electrolyte disorders & 548 & 0.78 & 656 & 1.16 \\
\hline Blood loss anaemia & 33 & 0.05 & 49 & 0.09 \\
\hline Deficiency anaemia & 537 & 0.76 & 773 & 1.37 \\
\hline Alcohol abuse & 873 & 1.24 & 395 & 0.70 \\
\hline Drug abuse & 101 & 0.14 & 46 & 0.08 \\
\hline Psychoses & 168 & 0.24 & 128 & 0.23 \\
\hline \multirow[t]{2}{*}{ Depression } & 511 & 0.73 & 847 & 1.50 \\
\hline & \multicolumn{2}{|l|}{ ISD } & & \\
\hline Solid metastases & 257 & 0.4 & 385 & 0.7 \\
\hline Chronic pulmonary disorders & 3162 & 4.5 & 3541 & 6.3 \\
\hline Heart failure/cardiomyopathy & 2568 & 3.7 & 1663 & 2.9 \\
\hline Malignancies & 2593 & 3.7 & 2464 & 4.4 \\
\hline Pulmonary circulation disorders & 141 & 0.2 & 163 & 0.3 \\
\hline Peripheral vascular disease & 1426 & 2.0 & 783 & 1.4 \\
\hline AIDS/HIV* & 15 & 0.0 & 5 & 0.0 \\
\hline Cerebrovascular disease & 968 & 1.4 & 783 & 1.4 \\
\hline Ischaemic heart disease & 7870 & 11.2 & 4748 & 8.4 \\
\hline Diabetes & 2664 & 3.8 & 2062 & 3.7 \\
\hline Liver disease & 935 & 1.3 & 655 & 1.2 \\
\hline Hypertension, complicated & 134 & 0.2 & 141 & 0.2 \\
\hline Cardiac arrhythmias & 3538 & 5.0 & 2819 & 5.0 \\
\hline Dementia & 206 & 0.3 & 354 & 0.6 \\
\hline Obesity & 1307 & 1.9 & 1353 & 2.4 \\
\hline Valvular heart disease & 787 & 1.1 & 765 & 1.4 \\
\hline Coagulopathy & 227 & 0.3 & 170 & 0.3 \\
\hline Drug/alcohol abuse & 2532 & 3.6 & 1104 & 2.0 \\
\hline Hemiplegia/paraplegia & 334 & 0.5 & 243 & 0.4 \\
\hline Other neurological disorders & 568 & 0.8 & 538 & 1.0 \\
\hline Renal disease & 782 & 1.1 & 627 & 1.1 \\
\hline
\end{tabular}


Table 2 Continued

\begin{tabular}{|c|c|c|c|c|}
\hline \multirow[b]{3}{*}{ Comorbidity } & \multicolumn{4}{|c|}{ Elixhauser } \\
\hline & \multicolumn{2}{|l|}{ Men } & \multicolumn{2}{|c|}{ Women } \\
\hline & $\mathbf{n}$ & Per cent & $\mathrm{N}$ & Per cent \\
\hline Nutritional anaemia & 560 & 0.8 & 809 & 1.4 \\
\hline Hypertension, uncomplicated & 7771 & 11.1 & 7341 & 13.0 \\
\hline Psychoses & 226 & 0.3 & 206 & 0.4 \\
\hline Malnutrition/weight loss & 251 & 0.4 & 224 & 0.4 \\
\hline
\end{tabular}

whether social mortality differentials in a diabetic population actually reflect variation in the overall burden of disease across socioeconomic strata. The findings of this study suggest that even after adjustment has been made for variation in the burden of non-diabetic disease, a residual effect of SES remains significantly predictive of mortality. The OR estimates representing the association between SIMD quintile and death are modestly attenuated when further adjustment is made for measures of comorbidity based on hospital records or primary care prescribing (figures 1 and 2). However, the basic structure of the association (loosely, a monotonic gradient in men and a contrast of 'most deprived vs others' in women) is broadly preserved after the introduction of comorbidity. This holds true irrespective of which measure of comorbidity derived from routine data is used. In passing, it merits highlighting that the between-sex difference in the pattern of association between SES and mortality evident in figures 1 and 2 is not consistent with the findings of Roper et al, ${ }^{5}$ who observed a gradient effect for both sexes.

On this basis of the study reported here, it may reasonably be concluded that SES-related mortality differences in a population with T2DM are not due to more affluent groups being 'less sick': some other dimensions of SES are implicated. Accepted influences on the social patterning of health and mortality include material deprivation, childhood social development, social exclusion, occupational status and security, educational attainment, and housing environment and tenure. ${ }^{24}{ }^{25}$ It is of course impossible to determine which (if any) of these may be

Table 3 Summary values for $\mathrm{CCl}$, number of in-patient bed-days, and number of unique BNF codes in patient's medication history

\begin{tabular}{lcc}
\hline & Men & Women \\
\hline CCI (n/\% of total) & & \\
0 & $56537 / 80.5$ & $45383 / 80.4$ \\
1 & $7321 / 10.4$ & $5983 / 10.6$ \\
$2-3$ & $5186 / 7.4$ & $4154 / 7.4$ \\
$4+$ & $1153 / 1.6$ & $931 / 1.6$ \\
Number of in-patient bed-days (n/\% of total) & & \\
0 & $35359 / 50.4$ & $26305 / 46.6$ \\
$1-4$ & $16400 / 23.4$ & $13208 / 23.4$ \\
$5-10$ & $7709 / 11.0$ & $6770 / 12.0$ \\
$\geq 11$ & $10729 / 15.3$ & $10168 / 18.0$ \\
Number of unique BNF codes & & \\
Minimum/maximum & $0 / 67$ & $0 / 67$ \\
Mean/SD & $9.4 / 7.4$ & $13.2 / 9.0$ \\
Median/lQR & $8 / 9$ & $12 / 11$ \\
n/\% of zero values (no comorbidity) & $5861 / 8.3$ & $3109 / 5.5$ \\
\hline BNF, British National Formulary; CCl, Charlson Comorbidity Index. &
\end{tabular}

$2 \begin{array}{llll}2 & 3 & 4 & 5\end{array}$

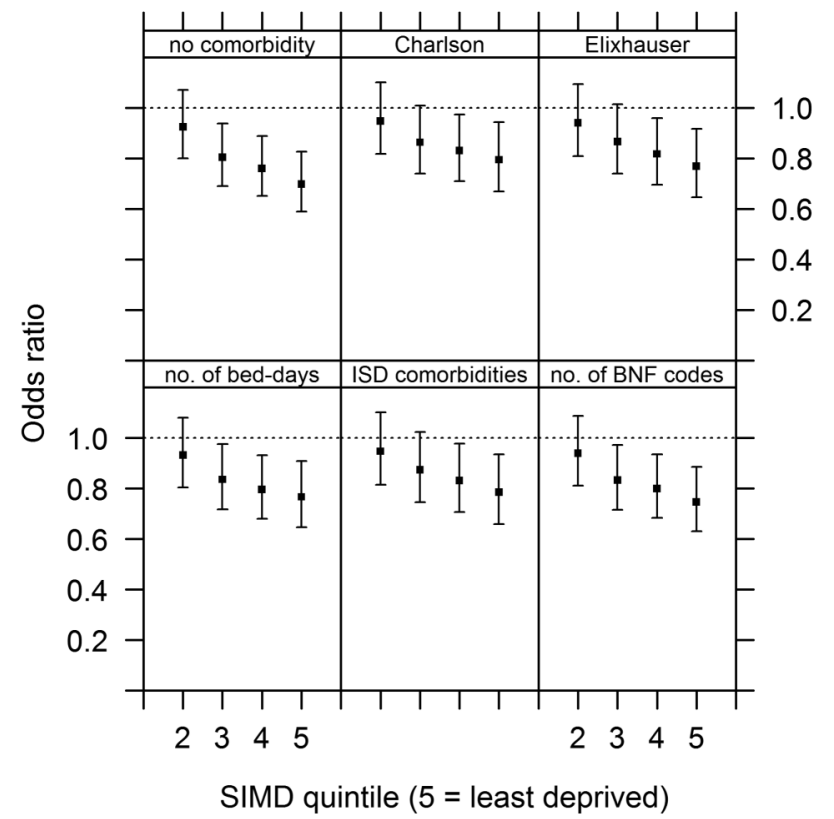

Figure 1 OR estimates for the association between socioeconomic status (Scottish Index of Multiple Deprivation (SIMD) quintile) and 1 -year mortality in men, adjusted for age only (no comorbidity) and for age plus five representations of comorbidity. Estimates are expressed relative to reference category SIMD quintile 1 (most deprived). Dashed horizontal line marks OR of unity ('no association').

contributing to the residual effect of SES (ie, that remaining after adjustment for comorbidity) in the population studied here.

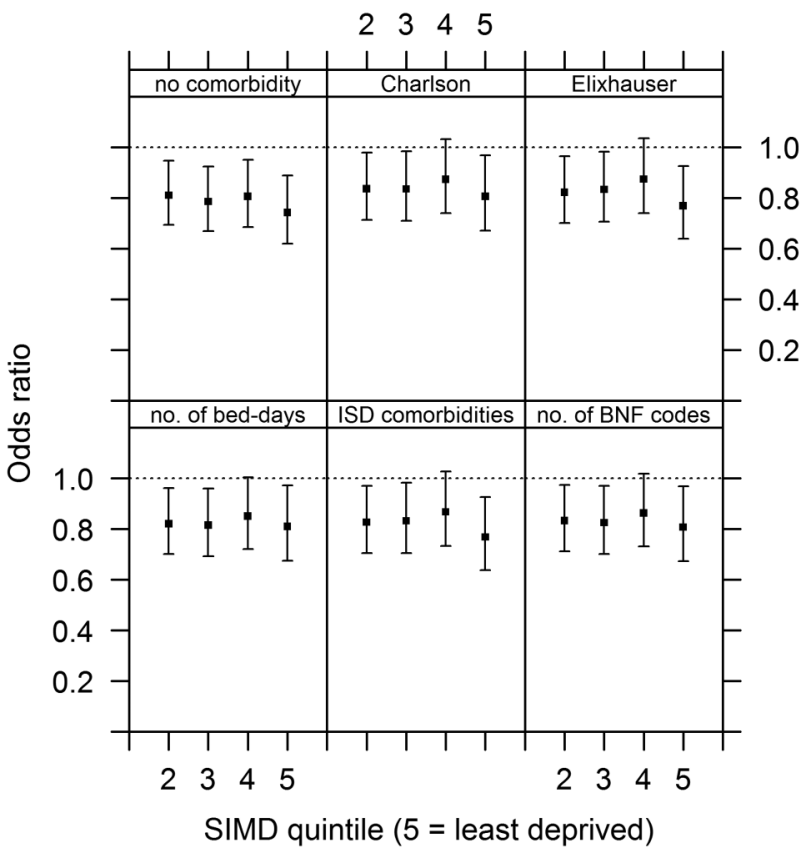

Figure 2 OR estimates for the association between socioeconomic status (Scottish Index of Multiple Deprivation (SIMD) quintile) and 1 -year mortality in women, adjusted for age only (no comorbidity) and for age plus five representations of comorbidity. Estimates are expressed relative to reference category SIMD quintile 1 (most deprived). Dashed horizontal line marks OR of unity ('no association'). 
Strengths of this study are threefold. First, it is populationbased: the data include almost all eligible individuals with diabetes in the entire Scottish population during the period covered. Consequently, many of the biases potentially introduced when research is based on selected samples (eg, patients from a specific group of hospitals) are avoided. A second strength of this investigation is the adoption of multiple methods of measuring comorbidity. As discussed earlier, there is no 'gold standard' for quantifying comorbidity (though the CCI has arguably become something of a de facto standard in this field), so it is advisable in a study such as the present to include on a comparative basis several different representations of comorbid disease. This provides protection against the findings reflecting the peculiarities of a specific comorbidity index. A third strength is the nature of the representation of SES used in the study. The SIMD was designed to provide a multifaceted portrayal of deprivation status, and thus arguably provides a better approximation to the individual's 'true' position in the social and economic hierarchy than single-dimension measures such as income or educational attainment.

Several limitations of the study are acknowledged. First, although five different approaches to representing the abstract concept of 'comorbidity' have been compared, none of these can claim to be truly comprehensive or of unimpeachable accuracy. Four of the measures used are based on hospital discharge records, and thus cannot reflect clinical conditions identified or treated in primary care. For example, the prevalence of the Elixhauser condition 'obesity' derived from hospital data (table 2) is many times lower than the authoritative prevalence values reported in the Scottish Diabetes Survey. ${ }^{26}$ Moreover, inconsistencies across hospitals or inaccuracies may arise in the coding of conditions. To illustrate, table 2 reveals instances of reported diabetes in the study data even though the comorbidity measures are derived from hospital episodes recorded prior to formal diagnosis of diabetes. While the fifth measure is constructed from medication records rather than hospital data, it also embodies a number of weaknesses. For example, it provides no indication of the intensity of drug treatment (ie, frequency and dosage), and obviously cannot reflect the patient's compliance with her/his medication regime (which might be expected to influence the outcome associated with the treatment). A second limitation of the study relates to the nature of the measure of SES used, that is, SIMD. As outlined above, this attempts to reflect the multidimensional nature of deprivation, but the use of an area-based measure to represent the characteristics of an individual leads to an obvious inferential pitfall: not all deprived people live in deprived areas, and not all residents of a deprived area are necessarily deprived. However, the 6505 areas used by SIMD (data zones) are relatively small in population terms, the average number of residents being c. 800 . Consequently, it may reasonably be assumed that the social makeup of each area is relatively homogeneous, with few instances of sizeable 'deprived' enclaves in affluent areas, and vice versa. Thus, the characteristics of the area should generally provide a reliable representation of individual circumstances. It merits highlighting that there is a very strong tradition of using SIMD to portray individual SES in health-related research performed in Scotland. Moreover, there is a growing body of international literature reporting the use of area-based measures to assess inequalities in health and mortality. ${ }^{27} \mathrm{~A}$ third limitation of the study is that we have not attempted to classify the severity of the patient's diabetes at the point of diagnosis. This is a potentially serious weakness, in that poor control and the presence of complications of diabetes would be expected to exhibit a stronger association with mortality than less serious manifestations of the disease. It cannot be discounted that severity of T2DM at presentation may vary by SES, reflecting socially patterned differences in healthcare-seeking behaviour. A fourth limitation is that because all measures of comorbidity were based on the patient's experience prior to the diagnosis of T2DM, no account is taken of the effect on mortality of complications of diabetes recorded subsequent to diagnosis (notably macrovascular disease, which is the main contributor to the excess mortality in people with diabetes). ${ }^{1}$ Another potentially important prognostic factor not taken into account is smoking behaviour. The prevalence of smoking is known to vary by SES, and previous research has indicated that both current and former smoking is associated with increased mortality in diabetic cohorts. ${ }^{28-30}$

Despite its limitations, this study suggests that the frequently observed phenomenon of SES-related mortality in people with diabetes is not wholly explained by a greater burden of disease in more deprived subgroups. However, the study was conducted in a Scottish population with T2DM, and this conclusion will not necessarily hold in other populations. Further research is required to determine the mechanisms via which the risk of death in people with diabetes, as in many other populations, is linked to SES. Of particular interest is the question of whether these mechanisms-whatever they may be-differ between people with diabetes and the general population, and whether it is possible to address these factors in order to reduce health inequalities.

\section{What is already known on this subject}

Mortality in people with diabetes - as in many other populations-is socially patterned, all-cause mortality risk being raised in more deprived groups.

\section{What this study adds}

- Socially patterned mortality in a population with type 2 diabetes is not explained by differential comorbidity (ie, greater overall burden of disease in deprived groups).

- This applies irrespective of how comorbidity is measured.

- Further research is needed to clarify how mortality in populations with diabetes is linked to deprivation.

Acknowledgements These data are available for analysis by members of the Scottish Diabetes Research Network epidemiology group; thanks to the hard work of numerous NHS staff who enter the data and people and organisations (the Scottish Care Information-Diabetes Collaboration (SCI-DC) Steering Group, the Scottish Diabetes Group, the Scottish Diabetes Survey Monitoring Group, the managed clinical network managers and staff in each Health Board) involved in setting up, maintaining and overseeing SCI-DC. Thanks are due also to staff of the Scottish Cancer Registry and to the ISD record linkage team. Contributions from the Advisory Group established to guide the study are greatly appreciated: the Group includes (in addition to the authors) Dr Corrie Black, Dr David Brewster, Professor Bruce Guthrie, and Mr Hugh Paterson.

Contributors SHW, NH, DM and CJW conceived and designed the study. JW analysed the data. JW wrote initial drafts of the paper. All authors made substantial contributions to revisions of the manuscript.

Funding The study was funded by the Scottish Government Chief Scientist Office (grant reference $\mathrm{CZH} / 4 / 922$ ).

Competing interests None declared. 
Ethical approval Use of SCI-DC and associated linked data for the purposes of this study was approved by the responsible ethics committee, Caldicott guardians and the NHSNSS Privacy Application Committee (application no. 33/11).

Provenance and peer review Not commissioned; externally peer reviewed.

Open Access This is an Open Access article distributed in accordance with the Creative Commons Attribution Non Commercial (CC BY-NC 4.0) license, which permits others to distribute, remix, adapt, build upon this work non-commercially, and license their derivative works on different terms, provided the original work is properly cited and the use is non-commercial. See: http://creativecommons.org/ licenses/by-nc/4.0/

\section{REFERENCES}

1 Nwaneri C, Cooper H, Bowen-Jones D. Mortality in type 2 diabetes mellitus: magnitude of the evidence from a systematic review and meta-analysis. $\mathrm{Br} \mathrm{J}$ Diabetes Vasc Dis 2013;13:192-207.

2 Hu FB, Stampfer MJ, Solomon CG, et al. The impact of diabetes mellitus on mortality from all causes and coronary heart disease in women. Arch Intern Med 2001;161:1717-23.

3 Lotufo PA, Gaziano JM, Chae CU, et al. Diabetes and all-cause and coronary heart disease mortality among US male physicians. Arch Intern Med 2001;161:242-7.

4 Robinson N, Lloyd CE, Stevens LK. Social deprivation and mortality in adults with diabetes mellitus. Diabet Med 1998;15:205-12.

5 Roper NA, Bilous RW, Kelly WF, et al. Excess mortality in a population with diabetes and the impact of material deprivation: longitudinal, population based study. BMJ 2001;322:1389-93.

6 Dray-Spira R, Gary-Webb TL, Brancati FL. Educational disparities in mortality among adults with diabetes in the U.S. Diabetes Care 2010;33:1200-5.

7 Charlson ME, Pompei P, Ales KL, et al. A new method of classifying prognostic comorbidity in longitudinal studies: development and validation. J Chronic Dis 1987:40:373-83.

8 Elixhauser A, Steiner C, Harris DR, et al. Comorbidity measures for use with administrative data. Med Care 1998;36:8-27.

9 Parks RW, Bettschart V, Frame $S$, et al. Benefits of specialisation in the management of pancreatic cancer: results of a Scottish population-based study. $\mathrm{Br} J$ Cancer 2004;91:459-65.

10 Brilleman SL, Salisbury C. Comparing measures of multimorbidity to predict outcomes in primary care: a cross sectional study. Fam Pract 2013;30:172-8.

11 Rochon PA, Katz JN, Morrow LA, et al. Comorbid illness is associated with survival and length of hospital stay in patients with chronic disability. Med Care 1996;34:1093-101.

12 Perkins AJ, Kroenke K, Unützer J, et al. Common comorbidity scales were similar in their ability to predict health care costs and mortality. J Clin Epidemiol 2004;57:1040-8.

13 Quail JM, Lix LM, Osman BA, et al. Comparing comorbidity measures for predicting mortality and hospitalization in three population-based cohorts. BMC Health Serv Res 2011;11:146.
14 McKnight JA, Morris AD, Cline D, et al. Implementing a national quality assurance system for diabetes care: the Scottish Diabetes Survey 2001-2006. Diabet Med 2008;25:743-6.

15 World Health Organization. International Statistical Classification of Diseases and Related Health Problems. 10th Revision. http://apps.who.int/classifications/icd10/ browse/2015/en (accessed 18 Sep 2015).

16 Romano PS, Roos LL, Jollis JG. Presentation adapting a clinical comorbidity index for use with ICD-9-CM administrative data: differing perspectives. J Clin Epidemiol 1993;46:1075-9.

17 NHS National Services Scotland. Hospital Standardised Mortality Ratios: Quarterly HSMR Release, 26 February 2013. http://www.isdscotland.org/Health-Topics/ Quality-Improvement/Publications/2013-02-26/2013-02-26-HSMR-Report.pdf (accessed 18 Sep 2015).

18 Quan $\mathrm{H}$, Sundararajan V, Halfon $\mathrm{P}$, et al. Coding algorithms for defining comorbidities in ICD-9-CM and ICD-10 administrative data. Med Care 2005;43:1130-9.

19 NHS National Services Scotland. Coding Guidelines, No. 21 (November 2007). http://www.isdscotland.org/Products-and-Services/Terminology-Services/ Clinical-Coding-Guidelines/Docs/Coding\%20Guidelines\%20No.\%2021,\% 20November\%202007.pdf (accessed 18 Sep 2015).

20 Joint Formulary Committee. British National Formulary. 69th edn. London: British Medical Association and Royal Pharmaceutical Society of Great Britain, 2015.

21 Office of the Chief Statistician, The Scottish Government. Scottish Index of Multiple Deprivation: 2009 General Report. http://www.scotland.gov.uk/Resource/Doc/933/ 0115249.pdf (accessed 18 Sep 2015).

22 Heinze $G$, Schemper M. A solution to the problem of separation in logistic regression. Stat Med 2002;21:2409-19.

23 Bartley M. Health inequality: an introduction to theories, concepts and methods. Cambridge: Polity Press, 2004.

24 Frank J, Haw S. Best practice guidelines for monitoring socioeconomic inequalities in health status: lessons from Scotland. Milbank Q 2011;11:658-93.

25 Wadsworth MEJ. Health inequalities in the life course perspective. Soc Sci Med 1997:44:859-69.

26 Scottish Diabetes Survey Monitoring Group. Scottish Diabetes Survey 2014. 2015. http://www.diabetesinscotland.org.uk/Publications/SDS2014.pdf (accessed 18 Sep 2015).

27 Ralston K, Dundas R, Leyland AH. A comparison of the Scottish Index of Multiple Deprivation (SIMD) 2004 with the $2009+1$ SIMD: does choice of measure affect the interpretation of inequality in mortality? Int J Health Geogr 2014;13:27.

28 Al-Delaimy WK, Willett WC, Manson JE, et al. Smoking and mortality among women with type 2 diabetes: the Nurses' Health Study cohort. Diabetes Care 2001;24:2043-8.

29 Florkowski CM, Scott RS, Coope PA, et al. Predictors of mortality from type 2 diabetes mellitus in Canterbury, New Zealand: a ten-year cohort study. Diabetes Res Clin Pract 2001;53:113-20.

30 Katakura $\mathrm{M}$, Naka $\mathrm{M}$, Kondo $\mathrm{T}$, et al. Normal mortality in the elderly with diabetes under strict control: outcome of 6-year prospective study. Diabetes Res Clin Pract 2007;78:108-14. 